\title{
Morphology for Image Processing. Part $\mathbf{I}^{1}$
}

\author{
Hiroshi Yamazaki \\ Shinshu University \\ Nagano, Japan
}

\author{
Czesław Byliński \\ University of Białystok \\ Poland
}

\author{
Katsumi Wasaki \\ Shinshu University \\ Nagano, Japan
}

Summary. In this article we defined mathematical morphology image processing with set operations. First, we defined Minkowski set operations and proved their properties. Next, we defined basic image processing, dilation and erosion proving basic fact about them [5], [8].

MML identifier: MORPH_01, version: $\underline{7.12 .02 \quad 4.170 .1134}$

The terminology and notation used in this paper have been introduced in the following papers: [10], [7], [1], [2], [6], [9], [4], and [3].

\section{Minkowski Set Operations}

Let $E$ be a non empty RLS structure. A binary image of $E$ is a subset of $E$. In the sequel $E$ denotes a real linear space and $A$ denotes a binary image of $E$. Let $E$ be a real linear space and let $A, B$ be binary images of $E$. The functor $A \ominus B$ yielding a binary image of $E$ is defined as follows:

(Def. 1) $A \ominus B=\left\{z \in E: \bigwedge_{b \text { : element of } E}(b \in B \Rightarrow z-b \in A)\right\}$.

Let $a$ be a real number, let $E$ be a real linear space, and let $A$ be a subset of $E$. We introduce $a \cdot A$ as a synonym of $a \odot A$. The following propositions are true:

(1) Let $E$ be a real linear space and $A, B$ be subsets of $E$. If $B=\emptyset$, then $A \oplus B=B$ and $B \oplus A=B$ and $A \ominus B=$ the carrier of $E$.

(2) For every real linear space $E$ and for all subsets $A, B$ of $E$ such that $A \neq \emptyset$ and $B=\emptyset$ holds $B \ominus A=B$.

\footnotetext{
${ }^{1}$ The authors wants to thank Prof. Yasunari Shidama for his kind support during the course of this work.

(C) 2012 University of Białystok CC-BY-SA License ver. 3.0 or later ISSN 1426-2630(p), 1898-9934(e)
} 
(3) Let $E$ be a real linear space and $A, B$ be subsets of $E$. If $B=$ the carrier of $E$ and $A \neq \emptyset$, then $A \oplus B=B$ and $B \oplus A=B$.

(4) For every real linear space $E$ and for all subsets $A, B$ of $E$ such that $B=$ the carrier of $E$ holds $B \ominus A=B$.

(5) $A \oplus B=\bigcup\{b+A ; b$ ranges over elements of $E: b \in B\}$.

Let $E$ be a non empty RLS structure. A binary image family of $E$ is a family of subsets of the carrier of $E$.

We follow the rules: $F, G$ are binary image families of $E$ and $A, B, C$ are non empty binary images of $E$. We now state four propositions:

(6) $A \ominus B=\bigcap\{b+A ; b$ ranges over elements of $E: b \in B\}$.

(7) $A \oplus B=\{v \in E:(v+(-1) \cdot B) \cap A \neq \emptyset\}$.

(8) $A \ominus B=\{v \in E: v+(-1) \cdot B \subseteq A\}$.

(9) ((The carrier of $E) \backslash A) \oplus B=$ (the carrier of $E) \backslash A \ominus B$ and ((the carrier of $E) \backslash A) \ominus B=($ the carrier of $E) \backslash A \oplus B$.

Let $E$ be a non empty Abelian additive loop structure and let $A, B$ be subsets of $E$. Let us note that the functor $A \oplus B$ is commutative.

One can prove the following propositions:

(10) For every non empty add-associative additive loop structure $E$ and for all subsets $A, B, C$ of $E$ holds $(A+B)+C=A+(B+C)$.

(11) $(A \oplus B) \oplus C=A \oplus(B \oplus C)$.

(12) $\bigcup F \oplus B=\bigcup\{X \oplus B ; X$ ranges over binary images of $E: X \in F\}$.

(13) $A \oplus \bigcup F=\bigcup\{A \oplus X ; X$ ranges over binary images of $E: X \in F\}$.

(14) $\bigcap F \oplus B \subseteq \bigcap\{X \oplus B ; X$ ranges over binary images of $E: X \in F\}$.

(15) $A \oplus \bigcap F \subseteq \bigcap\{A \oplus X ; X$ ranges over binary images of $E: X \in F\}$.

(16) For every non empty additive loop structure $E$ and for all subsets $A, B$, $C$ of $E$ such that $B \subseteq C$ holds $A+B \subseteq A+C$.

(17) $(v+A) \oplus B=A \oplus(v+B)$ and $(v+A) \oplus B=v+A \oplus B$.

(18) $\bigcap F \ominus B=\bigcap\{X \ominus B ; X$ ranges over binary images of $E: X \in F\}$.

(19) $\bigcap\{B \ominus X ; X$ ranges over binary images of $E: X \in F\} \subseteq B \ominus \bigcap F$.

(20) $\bigcup\{X \ominus B ; X$ ranges over binary images of $E: X \in F\} \subseteq \bigcup F \ominus B$.

(21) If $F \neq \emptyset$, then $B \ominus \bigcup F=\bigcap\{B \ominus X ; X$ ranges over binary images of $E$ : $X \in F\}$.

(22) If $A \subseteq B$, then $A \ominus C \subseteq B \ominus C$.

(23) If $A \subseteq B$, then $C \ominus B \subseteq C \ominus A$.

(24) $(v+A) \ominus B=A \ominus(v+B)$ and $(v+A) \ominus B=v+A \ominus B$.

(25) $A \ominus B \ominus C=A \ominus(B \oplus C)$. 


\section{Dilation AND Erosion}

Let $E$ be a real linear space and let $B$ be a binary image of $E$. The functor

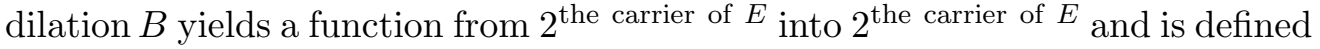
as follows:

(Def. 2) For every binary image $A$ of $E$ holds (dilation $B)(A)=A \oplus B$.

Let $E$ be a real linear space and let $B$ be a binary image of $E$. The functor erosion $B$ yields a function from $2^{\text {the carrier of } E}$ into $2^{\text {the carrier of } E}$ and is defined by:

(Def. 3) For every binary image $A$ of $E$ holds (erosion $B)(A)=A \ominus B$.

The following propositions are true:

(26) (dilation $B)(\bigcup F)=\bigcup\{($ dilation $B)(X) ; X$ ranges over binary images of $E: X \in F\}$.

(27) If $A \subseteq B$, then $($ dilation $C)(A) \subseteq($ dilation $C)(B)$.

(28) $($ dilation $C)(v+A)=v+(\operatorname{dilation} C)(A)$.

(29) (erosion $B)(\bigcap F)=\bigcap\{($ erosion $B)(X) ; X$ ranges over binary images of $E: X \in F\}$.

(30) If $A \subseteq B$, then $(\operatorname{erosion} C)(A) \subseteq(\operatorname{erosion} C)(B)$.

(31) $(\operatorname{erosion} C)(v+A)=v+(\operatorname{erosion} C)(A)$.

(32) (dilation $C)(($ the carrier of $E) \backslash A)=($ the carrier of $E) \backslash(\operatorname{erosion} C)(A)$ and (erosion $C)(($ the carrier of $E) \backslash A)=($ the carrier of $E) \backslash($ dilation $C)(A)$.

(33) $\quad($ dilation $C)(($ dilation $B)(A))=($ dilation $($ dilation $C)(B))(A)$ and $(\operatorname{erosion} C)((\operatorname{erosion} B)(A))=(\operatorname{erosion}($ dilation $C)(B))(A)$.

\section{REFERENCES}

[1] Czesław Byliński. Functions and their basic properties. Formalized Mathematics, 1(1):5565, 1990.

[2] Czesław Byliński. Functions from a set to a set. Formalized Mathematics, 1(1):153-164, 1990.

[3] Yuzhong Ding and Xiquan Liang. Preliminaries to mathematical morphology and its properties. Formalized Mathematics, 13(2):221-225, 2005.

[4] Noboru Endou, Takashi Mitsuishi, and Yasunari Shidama. Dimension of real unitary space. Formalized Mathematics, 11(1):23-28, 2003.

[5] H.J.A.M. Heijimans. Morphological Image Operators. Academic Press, 1994.

[6] Krzysztof Hryniewiecki. Basic properties of real numbers. Formalized Mathematics, 1(1):35-40, 1990.

[7] Beata Padlewska. Families of sets. Formalized Mathematics, 1(1):147-152, 1990.

[8] P. Soille. Morphological Image Analysis: Principles and Applications. Springer, 2003.

[9] Wojciech A. Trybulec. Vectors in real linear space. Formalized Mathematics, 1(2):291-296, 1990.

[10] Zinaida Trybulec. Properties of subsets. Formalized Mathematics, 1(1):67-71, 1990.

Received September 21, 2011 